\title{
Propiedades materiales e índices de rendimiento acústico de veintidós maderas mexicanas. Determinación por ultrasonido
}

\section{Material properties and acoustic performance indexes of twenty-two Mexican woods. Measurement by ultrasound}

\author{
Javier Ramón Sotomayor Castellanos ${ }^{* *}$ y José María Villaseñor Aguilar
}

\begin{abstract}
Universidad Michoacana de San Nicolás de Hidalgo. Facultad de Ingeniería en Tecnología de la Madera.

Morelia, Michoacán, México.
\end{abstract}

\section{RESUMEN}

El objetivo de la investigación fue determinar las propiedades materiales (densidad aparente, velocidad del ultrasonido, módulo dinámico y coeficiente de amortiguamiento) y los índices de rendimiento acústico (coeficiente de radiación acústica, coeficiente de eficiencia acústica, índice de vibración acústica e índice de anti-vibración acústica) de la madera de 22 especies mexicanas, con el fin de evaluar su potencial uso en aplicaciones acústicas, como lo es la fabricación de instrumentos musicales en general, y en particular, los de cuerda. Se encontró que las propiedades materiales y los índices de rendimiento acústico de las veintidós maderas estudiadas, son características de cada una de ellas. La velocidad del ultrasonido no presenta una relación lineal con la densidad aparente de la madera. En cambio, el módulo dinámico es proporcional a la densidad aparente y las maderas de baja densidad aparente presentan mejores coeficientes de eficiencia acústica en comparación con las especies de altas densidades. Se concluye que un criterio de selección para una madera con aptitudes para ser utilizada en la fabricación de instrumentos musicales es la combinación de una baja densidad aparente, un alto módulo dinámico y un bajo coeficiente de amortiguamiento.

PALABRAS CLAVE: densidad aparente, coeficiente de amortiguamiento, instrumentos musicales, módulo dinámico, velocidad del ultrasonido.

\section{ABSTRACT}

The goal of the research was to evaluate the material properties (apparent density, ultrasound speed, dynamic modulus and damping coefficient) and the indexes of the acoustic performance (acoustic radiation coefficient, acoustic impedance, efficiency acoustic coefficient, acoustic radiation coefficient, acoustic vibration index and anti-vibration acoustic index) of twenty-two Mexican woods with the purpose of evaluating its potential use in acoustic applications, such as the manufacturing of musical instruments in general, and string instruments, in particular. It was found that the material properties and the indexes of the acoustic performance of twenty-two woods studied are characteristics specific to each one of them. The speed of the ultrasound does not have a linear relationship with the apparent density of the wood. In contrast, the dynamic modulus is proportional to the apparent density. Low density woods have better acoustic efficiency coefficients compared to high density species. A selection criterion for a wood with aptitudes to be used in the manufacture of musical instruments is the combination of a low bulk density, a high dynamic modulus and a low damping coefficient.

KEYWORDS: apparent density, damping coefficient, musical instruments, dynamic modulus, ultrasound speed. 


\section{INTRODUCCIÓN}

El empleo de la madera en la manufactura de instrumentos musicales de cuerda, de viento y de percusión es una de las más altas valoraciones de utilización del material, particularmente las especies de maderas con óptimas propiedades acústicas, calificadas como "madera de resonancia" (Bucur, 2006). Las cualidades de la madera relacionadas con su caracterización acústica son importantes también para su valoración como materia prima para la elaboración de amplificadores de sonido y de cajas musicales de resonancia.

En México, existen maderas con características acústicas apropiadas para su uso en instrumentos musicales. Sin embargo, los fabricantes no cuentan con datos o indicadores que evalúen de una manera cuantitativa las propiedades acústicas y de calidad de las maderas utilizadas. Los productores de instrumentos musicales seleccionan las especies nacionales e importadas de acuerdo con su experiencia y con el precio de las maderas accesibles en el mercado. Incluso, la tecnología de producción y la determinación de la calidad de los productos se basan principalmente en métodos empíricos.

En la literatura científica, la caracterización del comportamiento acústico de maderas empleadas tradicionalmente y con potencial para la elaboración de instrumentos musicales puede ser consultada, entre otros, en Ilic (2003), Bucur (2006), Spycher, Schwarze y Steiger (2008), Sedik, Hamdan, Jusoh y Hasan (2010), Brémaud et al. (2012) y Fouilhé, Houssay y Brémaud (2012). Estos autores determinan las principales características y los índices de calidad acústicos de maderas en el ámbito internacional.

Las propiedades acústicas de la madera y su relación con sus características anatómicas han sido estudiadas exhaustivamente por Ono y Norimoto (1983; 1984; 1985), Obataya, Ono, y Norimoto (2000), Brancheriau, Baillères, Détienne, Gril y Kronland (2006), Wegst (2006), Yoshikawa (2007), Smardzewski et al. (2014), Traoré, Loïc, Perré, Stevanovic y Diouf (2010), Roohnia, Tajdini y Manouchehri (2011), Fouilhé et al. (2012) y Yoshikawa y Waltham (2014). Las investigaciones citadas también identifican categorías de instrumentos musicales donde la madera se incorpora como elemento estético, de soporte estructural, como placa o caja de resonancia y como componente en la dimensión cultural. Entre otras categorías, se mencionan instrumentos de cuerda, de viento y de percusión donde la madera es utilizada.

Las características tecnológicas de maderas mexicanas están documentadas, entre otros, por Tamarit y López (2007) y Silva et al. (2010). Sin embargo, sobre el tema de características acústicas e indicadores relacionados con la calidad de la madera, la revisión de la bibliografía del país exhibe poca información al respecto. Sotomayor, Guridi y García (2010) presentan resultados experimentales de las propiedades acústicas y sobre la calidad de maderas mexicanas con uso y potencial para elaborar instrumentos musicales.

Otros autores proponen maderas mexicanas para su estudio y promoción en aplicaciones acústicas: Barajas y León (1989), Guridi y García (1997), Gutiérrez y Dorantes (2007), Tamarit y López (2007) y Silva et al. (2010). Estos investigadores, no presentan indicadores técnicos relacionados explícitamente con el comportamiento acústico de la madera. Para recomendar el uso de especies mexicanas, los trabajos citados parten del análisis de la estructura anatómica de las especies, así como de indicadores tecnológicos. Como complemento, los autores citados retoman la opinión de fabricantes de instrumentos musicales, sobre la pertinencia de una madera para su uso en instrumentos musicales.

Este enfoque de clasificación de una madera para su recomendación en la elaboración de instrumentos musicales, es de carácter práctico y encuentra sustento en que, para la selección de una madera, el fabricante se guía preferentemente por indicadores visuales, que son expresión de la estructura macroanatómica de una especie en particular (Buksnowitz, Teischinger, Müller, Pahler y Evans, 2007). Además, el valor cultural que se la da a cada instrumento es factor importante en el diseño (Brémaud, 2012a; Fouilhé et al. 2012).

Efectivamente, la selección de una madera para su empleo en un instrumento musical tiene que comple- 
mentarse con la apreciación de fabricantes y usuarios de instrumentos musicales. Sin olvidar también que, para optimizar el rendimiento de un componente de un instrumento musical, son necesarios procesos de fabricación y de acabado, propios para la función del componente y para el tipo de instrumento en cuestión (Bucur, 2006).

El sonido producido por una pieza de madera, es resultado de la energía ocasionada por sus vibraciones, transmitidas en un medio físico. De aquí, que el rendimiento acústico de una madera en particular, depende de sus propiedades materiales, las cuales determinan las frecuencias y velocidades de onda propias a una pieza de madera, con una masa y una geometría determinadas. El sonido se puede definir como la vibración mecánica de un medio elástico en el cual se irradia energía en forma de ondas acústicas (Smardzewski, 2014). En el caso de la madera, el sonido se transmite por medio de los líquidos contenidos en los lúmenes y paredes celulares y a través de la matriz lignocelulósica, sólida y elástica, de la cual están formadas las paredes celulares del plano leñoso. Además, la regularidad de los anillos de crecimiento y la ausencia de nudos son, entre otros, atributos deseables para una madera con vocación musical (Spycher et al. 2008).

La velocidad de onda y la frecuencia de vibración de una barra sometida a flexión dinámica transversal, están relacionadas particularmente para el primer modo de vibración (Illic, 2003). En el mismo orden de ideas, la combinación de estas características con la densidad aparente resulta en la definición de indicadores y/o índices de rendimiento acústico (Roohnia et al. 2011 y Brémaud, 2012a). Desde otra perspectiva, la combinación de características acústicas y dimensiones de los componentes de un instrumento musical, por ejemplo el espesor de las placas de madera para cajas de resonancia, son los parámetros más importantes en el diseño de instrumentos musicales (Yoshikawa, 2007; Yoshikawa y Waltham, 2014).

Para una primera evaluación de la madera en aplicaciones acústicas, son necesarias tres características del material: su densidad aparente, su módulo dinámico y su coeficiente de amortiguamiento (Spycher et al., 2008) y un descriptor de la señal acústica como lo es la velocidad del ultrasonido (Brancheriau et al., 2006). Además, Roohnia et al. (2011) y Brémaud (2012a) proponen entre otros indicadores de la calidad acústica de la madera, los índices acústicos de rendimiento: coeficiente de radiación acústica y coeficiente de eficiencia acústica. Hay que considerar, además, que su estabilidad dimensional (Buksnowit et al., 2007) y la apreciación cultural de su especie (Fouilhé et al., 2012) son criterios necesarios para la selección de una madera.

Los índices acústicos de rendimiento explican las características de resonancia de una madera y son igualmente necesarios en el diseño de instrumentos musicales y en el de componentes donde las cualidades de aislamiento y/o resonancia acústica son deseables, por ejemplo, en el cálculo de elementos estructurales y /o arquitectónicos en edificaciones con madera (Dietsch y Köhler, 2010).

La ausencia o dificultad para encontrar información técnica estandarizada, que oriente al ingeniero y diseñador en la correcta utilización de especies maderables, es una razón para que la madera no sea considerada como un material de ingeniería confiable, y para impedir su utilización generalizada en productos con alto valor agregado, como es el caso de los instrumentos musicales.

\section{OBJETIVOS}

El objetivo de la investigación fue determinar las propiedades materiales: densidad aparente, velocidad del ultrasonido, módulo dinámico y coeficiente de amortiguamiento de la madera de 22 especies mexicanas y, a partir de ellas, los índices de rendimiento acústico: coeficiente de radiación acústica, coeficiente de eficiencia acústica, índice de vibración acústica e índice de antivibración acústica.

El alcance y la orientación de la investigación son el uso de la madera en aplicaciones acústicas, como la fabricación de instrumentos musicales en general, y en particular, los de cuerda. Sin embargo, los resultados probablemente pueden utilizarse, con los debidos ajustes de cada caso, en el diseño y cálculo de productos y estructuras de madera. 


\section{MATERIALES Y MÉTODOS}

El material experimental consistió en maderas de 22 especies, recolectadas en terrenos forestales del estado de Michoacán, México. Los taxones botánicos de las especies fueron identificados en la Facultad de Ingeniería en Tecnología de la Madera, de la Universidad Michoacana de San Nicolás de Hidalgo, Morelia, Michoacán, México. La tabla 1 presenta la lista de especies en estudio. Para cada especie, se prepararon 20 probetas con dimensiones de 50 $\mathrm{mm} \times 50 \mathrm{~mm}$ en su sección transversal y con longitud entre $400 \mathrm{~mm}$ y $500 \mathrm{~mm}$. Las probetas estuvieron orientadas en las direcciones radial, tangencial y longitudinal del plano leñoso. La madera se estabilizó durante 24 meses en una cámara de acondicionamiento con una temperatura de 20 ${ }^{\circ} \mathrm{C}\left( \pm 1^{\circ} \mathrm{C}\right)$ y una humedad relativa de $60 \%( \pm 2 \%)$, hasta que alcanzó un contenido de humedad uniforme de $11 \%$. El contenido de humedad de la madera, se determinó por el método de diferencia de pesos (International Organization for Standardization [ISO], 2014), empleando grupos complementarios de 35 probetas para cada especie con dimensiones de $20 \mathrm{~mm} \times 20 \mathrm{~mm} \times 60 \mathrm{~mm}$.

Las pruebas de ultrasonido se realizaron aplicando un impulso ultrasónico de transmisión directa en la dirección longitudinal de la probeta. El tiempo de transmisión del

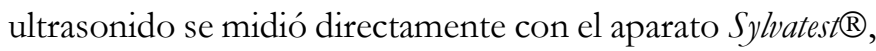
colocado en un dispositivo para pruebas no destructivas diseñado ad-hoc. Para esto, se colocaron los sensores emisor y receptor en los extremos de la probeta (Fig. 1). La velocidad del ultrasonido se calculó como el cociente de la longitud de las probetas entre el tiempo de transmisión. Los parámetros definidos a continuación tienen el subíndice "us", para significar que son parámetros derivados de pruebas de ultrasonido.

La densidad aparente de la madera al momento del ensayo se calculó con la fórmula (1):

$$
\varrho_{C H}=\frac{P_{C H}}{V_{C H}}
$$

donde:

$\varrho_{C H}=$ Densidad aparente de la madera a un contenido de humedad $\mathrm{CH}\left(\mathrm{kg} / \mathrm{m}^{3}\right)$
$P_{C H}=$ Peso de la probeta a un contenido de humedad $\mathrm{CH}$ $(\mathrm{kg})$

$V_{C H}=$ Volumen de la probeta a un contenido de humedad $\mathrm{CH}\left(\mathrm{m}^{3}\right)$

El módulo dinámico se calculó con la fórmula (2) propuesta por Brémaud (2012a):

$$
E_{u s}=v_{u s}^{2} \varrho_{C H}
$$

donde:

$E_{u s}=$ Módulo dinámico $\left(\mathrm{N} / \mathrm{m}^{2}\right)$

$v_{u s}=$ Velocidad del ultrasonido $(\mathrm{m} / \mathrm{s})$

$\varrho_{C H}=$ Densidad aparente de la probeta a un contenido de humedad $\mathrm{CH}\left(\mathrm{kg} / \mathrm{m}^{3}\right)$

El coeficiente de amortiguamiento se calculó con la fórmula

(3) propuesta por Brémaud et al. (2012):

$$
\delta_{u s}=10^{-1.322}\left(\frac{E_{u s}}{\varrho_{C H}}\right)^{-0.692}
$$

donde:

$\delta_{u s}=$ Coeficiente de amortiguamiento

$E_{u s}=$ Módulo dinámico $\left(\mathrm{N} / \mathrm{m}^{2}\right)$

$\varrho_{C H}=$ Densidad aparente de la madera a un contenido de humedad $\mathrm{CH}\left(\mathrm{kg} / \mathrm{m}^{3}\right)$

El coeficiente de radiación acústica se calculó de acuerdo con la fórmula (4) propuesta por Brémaud (2012a):

$$
\mathrm{R}_{u s}=\frac{v_{u s}}{\varrho_{C H}}
$$

donde:

$\mathrm{R}_{u s}=$ Coeficiente de radiación acústica $\left(\mathrm{m}^{4} / \mathrm{kg} \mathrm{s}\right)$

$v_{u s}=$ Velocidad del ultrasonido $(\mathrm{m} / \mathrm{s})$

$\varrho_{C H}=$ Densidad aparente de la madera a un contenido de humedad $\mathrm{CH}\left(\mathrm{kg} / \mathrm{m}^{3}\right)$

El coeficiente de eficiencia acústica se calculó con la fórmula (5) propuesta por Roohnia et al. (2011):

$$
C E A_{u s}=\frac{v_{u s}}{\varrho_{C H}{ }_{u s}}
$$


TABLA 1. Densidad aparente a $11 \%$ de $\mathrm{CH}$, velocidad del ultrasonido, módulo dinámico y coeficiente de amortiguamiento de la madera de 22 especies forestales del estado de Michoacán, México.

\begin{tabular}{|c|c|c|c|c|c|c|}
\hline No. & Especie & & $\begin{array}{c}\rho_{C H} \\
\left(\mathrm{~kg} / \mathrm{m}^{3}\right)\end{array}$ & $\begin{array}{c}V_{u s} \\
(\mathrm{~m} / \mathrm{s})\end{array}$ & $\begin{array}{c}E_{u s} \\
(\mathrm{MPa})\end{array}$ & $\begin{array}{c}\delta_{u s} \\
(\mathrm{~N} \mathrm{~m} / \mathrm{kg})\end{array}$ \\
\hline 1 & Spathodea campanulata & $\begin{array}{c}\bar{x} \\
C V\end{array}$ & $\begin{array}{l}338 \\
5.30\end{array}$ & $\begin{array}{c}3708 \\
6.84\end{array}$ & $\begin{array}{c}4646 \\
9.94\end{array}$ & $\begin{array}{c}0.0078 \\
-\end{array}$ \\
\hline 2 & Gyrocarpus americanus & $\begin{array}{c}\bar{x} \\
C V\end{array}$ & $\begin{array}{c}391 \\
3.49\end{array}$ & $\begin{array}{c}4488 \\
3.12\end{array}$ & $\begin{array}{l}7884 \\
6.50\end{array}$ & $\begin{array}{c}0.0060 \\
-\end{array}$ \\
\hline 3 & Tilia mexicana & $\begin{array}{c}\bar{x} \\
C V\end{array}$ & $\begin{array}{c}442 \\
12.55\end{array}$ & $\begin{array}{l}5260 \\
4.00\end{array}$ & $\begin{array}{c}12238 \\
13.31\end{array}$ & $\begin{array}{c}0.0049 \\
-\end{array}$ \\
\hline 4 & Enterolobium cyclocarpum & $\begin{array}{c}\bar{x} \\
C V\end{array}$ & $\begin{array}{l}448 \\
7.88\end{array}$ & $\begin{array}{c}3909 \\
4.09\end{array}$ & $\begin{array}{l}6845 \\
10.32\end{array}$ & $\begin{array}{c}0.0072 \\
-\end{array}$ \\
\hline 5 & Cupressus lindley & $\begin{array}{c}\bar{x} \\
C V\end{array}$ & $\begin{array}{c}486 \\
13.43\end{array}$ & $\begin{array}{c}4781 \\
10.92\end{array}$ & $\begin{array}{l}11111 \\
21.51\end{array}$ & $\begin{array}{c}0.0055 \\
-\end{array}$ \\
\hline 6 & Cedrela odorata & $\begin{array}{c}\bar{x} \\
C V\end{array}$ & $\begin{array}{c}517 \\
15.68\end{array}$ & $\begin{array}{l}4239 \\
10.16\end{array}$ & $\begin{array}{l}9226 \\
18.76\end{array}$ & $\begin{array}{c}0.0065 \\
-\end{array}$ \\
\hline 7 & Swietenia macrophylla & $\begin{array}{c}\bar{x} \\
C V\end{array}$ & $\begin{array}{l}531 \\
6.53\end{array}$ & $\begin{array}{c}4683 \\
6.91\end{array}$ & $\begin{array}{l}11674 \\
12.96\end{array}$ & $\begin{array}{c}0.0056 \\
-\end{array}$ \\
\hline 8 & Tabebuia donnell-smithii & $\begin{array}{c}\bar{x} \\
C V\end{array}$ & $\begin{array}{l}598 \\
3.62\end{array}$ & $\begin{array}{c}4306 \\
4.28\end{array}$ & $\begin{array}{c}11108 \\
9.33\end{array}$ & $\begin{array}{c}0.0064 \\
-\end{array}$ \\
\hline 9 & Dalbergia palo-escrito & $\begin{array}{c}\bar{x} \\
C V\end{array}$ & $\begin{array}{l}624 \\
7.68\end{array}$ & $\begin{array}{c}4697 \\
9.50\end{array}$ & $\begin{array}{l}13879 \\
21.26\end{array}$ & $\begin{array}{c}0.0056 \\
-\end{array}$ \\
\hline 10 & Tabeb & $\begin{array}{c}\bar{x} \\
C V\end{array}$ & $\begin{array}{l}635 \\
5.37\end{array}$ & $\begin{array}{c}4764 \\
5.37\end{array}$ & $\begin{array}{c}14421 \\
9.26\end{array}$ & $\begin{array}{c}0.0055 \\
-\end{array}$ \\
\hline 11 & Fagus mexicana & $\begin{array}{c}\bar{x} \\
C V\end{array}$ & $\begin{array}{l}642 \\
7.47\end{array}$ & $\begin{array}{c}4635 \\
4.22\end{array}$ & $\begin{array}{l}13854 \\
12.99\end{array}$ & $\begin{array}{c}0.0057 \\
-\end{array}$ \\
\hline 12 & Andira inermis & $\begin{array}{c}\bar{x} \\
C V\end{array}$ & $\begin{array}{l}716 \\
4.02\end{array}$ & $\begin{array}{l}4183 \\
4.49\end{array}$ & $\begin{array}{c}12528 \\
8.26\end{array}$ & $\begin{array}{c}0.0066 \\
-\end{array}$ \\
\hline 13 & Psidium sartorianum & $\begin{array}{c}\bar{x} \\
C V\end{array}$ & $\begin{array}{l}789 \\
3.58\end{array}$ & $\begin{array}{c}3935 \\
3.88\end{array}$ & $\begin{array}{c}12214 \\
6.73\end{array}$ & $\begin{array}{c}0.0072 \\
-\end{array}$ \\
\hline 14 & Juglans pyriformis & $\begin{array}{c}\bar{x} \\
C V\end{array}$ & $\begin{array}{l}810 \\
3.31\end{array}$ & $\begin{array}{c}4573 \\
5.63\end{array}$ & $\begin{array}{c}16999 \\
11.84\end{array}$ & $\begin{array}{c}0.0058 \\
-\end{array}$ \\
\hline 15 & Caesalpinia platyloba & $\begin{array}{c}\bar{x} \\
C V\end{array}$ & $\begin{array}{l}825 \\
2.59\end{array}$ & $\begin{array}{c}4853 \\
6.50\end{array}$ & $\begin{array}{c}19533 \\
14.73\end{array}$ & $\begin{array}{c}0.0053 \\
-\end{array}$ \\
\hline 16 & Albizia plurijuga & $\begin{array}{c}\bar{x} \\
C V\end{array}$ & $\begin{array}{l}844 \\
6.73\end{array}$ & $\begin{array}{c}4903 \\
2.93\end{array}$ & $\begin{array}{c}20254 \\
5.11\end{array}$ & $\begin{array}{c}0.0053 \\
-\end{array}$ \\
\hline 17 & Quercus spp. & $\begin{array}{c}\bar{x} \\
C V\end{array}$ & $\begin{array}{l}847 \\
3.46\end{array}$ & $\begin{array}{c}5256 \\
6.17\end{array}$ & $\begin{array}{c}23448 \\
11.65\end{array}$ & $\begin{array}{c}0.0048 \\
-\end{array}$ \\
\hline 18 & Lysiloma acapulcensis & $\begin{array}{c}\bar{x} \\
C V\end{array}$ & $\begin{array}{l}974 \\
3.72\end{array}$ & $\begin{array}{c}4576 \\
5.26\end{array}$ & $\begin{array}{c}20442 \\
10.35\end{array}$ & $\begin{array}{c}0.0058 \\
-\end{array}$ \\
\hline 19 & Cordia elaeagnoides & $\begin{array}{c}\bar{x} \\
C V\end{array}$ & $\begin{array}{l}992 \\
8.21\end{array}$ & $\begin{array}{c}4667 \\
4.72\end{array}$ & $\begin{array}{l}21610 \\
10.70\end{array}$ & $\begin{array}{c}0.0057 \\
-\end{array}$ \\
\hline 20 & Acosmium panamense & $\begin{array}{c}\bar{x} \\
C V\end{array}$ & $\begin{array}{c}1005 \\
6.17\end{array}$ & $\begin{array}{c}4550 \\
4.84\end{array}$ & $\begin{array}{c}20889 \\
12.26\end{array}$ & $\begin{array}{c}0.0058 \\
-\end{array}$ \\
\hline 21 & Tabebuia chrysantha & $\begin{array}{c}\bar{x} \\
C V\end{array}$ & $\begin{array}{c}1096 \\
2.23\end{array}$ & $\begin{array}{c}4427 \\
4.68\end{array}$ & $\begin{array}{c}21518 \\
9.84\end{array}$ & $\begin{array}{c}0.0061 \\
-\end{array}$ \\
\hline 22 & Dalbergia granadillo & $\begin{array}{c}\bar{x} \\
C V\end{array}$ & $\begin{array}{l}1147 \\
5.14 \\
\end{array}$ & $\begin{array}{c}4450 \\
6.18 \\
\end{array}$ & $\begin{array}{l}22821 \\
14.06 \\
\end{array}$ & $\begin{array}{c}0.0060 \\
- \\
\end{array}$ \\
\hline
\end{tabular}

$\rho_{C H}=$ Densidad aparente; $V_{u s}=$ Velocidad del ultrasonido; $E_{u s}=$ Módulo dinámico; $\delta_{u s}$ = Coeficiente de amortiguamiento; $\bar{x}=$ Media; $C V$ = Coeficiente de variación en porciento. 


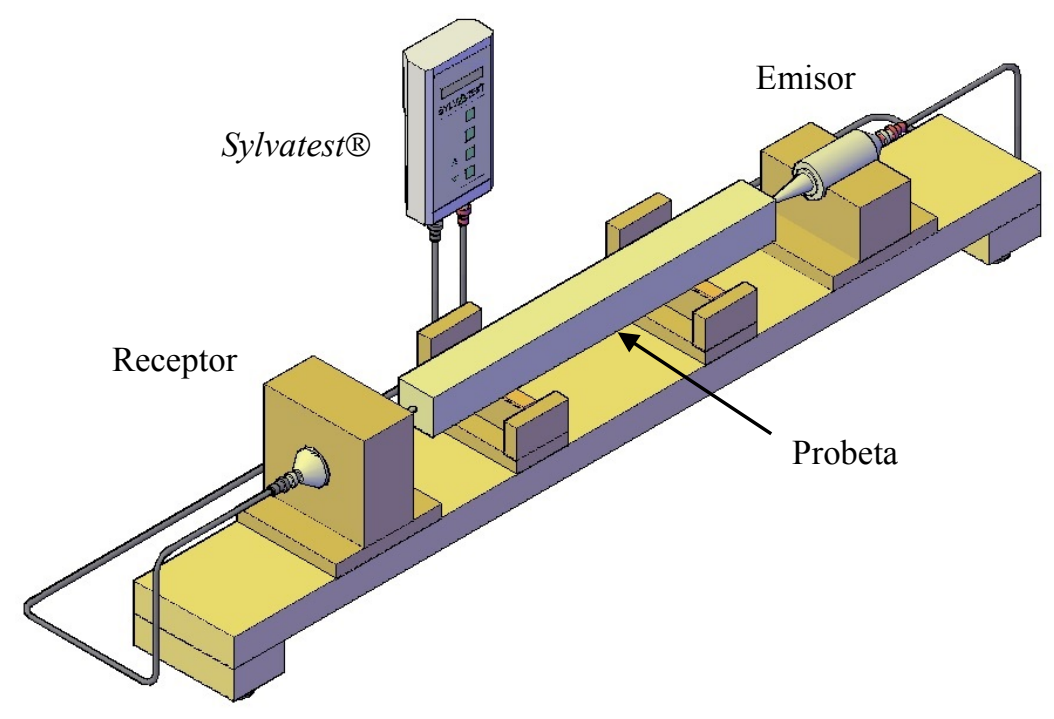

FIGURA 1. Dispositivo para las pruebas de ultrasonido.

donde:

$C E A_{u s}=$ Coeficiente de eficiencia acústica $(\mathrm{m} / \mathrm{s})$

$v_{u s}=$ Velocidad del ultrasonido $(\mathrm{m} / \mathrm{s})$

$\varrho_{C H}=$ Densidad aparente de la madera a un contenido de humedad $\mathrm{CH}\left(\mathrm{kg} / \mathrm{m}^{3}\right)$

$\delta_{u s}=$ Coeficiente de amortiguamiento (Sin dimensiones: $\mathrm{s} / \mathrm{d})$

El índice de vibración acústica se calculó con la fórmula (6) propuesta por Yoshikawa y Waltham (2014):

$$
I V A_{u s}=\frac{v_{u s}}{\delta_{u s}}
$$

donde:

$I V A_{u s} \quad=$ Índice de vibración acústica $(\mathrm{m} / \mathrm{s})$

$v_{u s}=$ Velocidad del ultrasonido $(\mathrm{m} / \mathrm{s})$

$\delta_{u s}=$ Coeficiente de amortiguamiento $(\mathrm{N} \mathrm{m} / \mathrm{kg})$

El índice de anti-vibración acústica se calculó con la fórmula (7) propuesta por Yoshikawa y Waltham (2014):

$$
I A A_{u s}=\frac{\varrho_{C H}}{v_{u s}}
$$

donde:

$L A A_{u s} \quad=$ Índice de anti-vibración acústica $\left(\mathrm{kg} \mathrm{s} / \mathrm{m}^{4}\right)$

$\varrho_{C H}=$ Densidad aparente de la madera a un contenido de humedad $\mathrm{CH}\left(\mathrm{kg} / \mathrm{m}^{3}\right)$

$v_{u s}=$ Velocidad del ultrasonido $(\mathrm{m} / \mathrm{s})$

\section{RESULTADOS Y DISCUSIÓN}

La tabla 1 presenta las densidades aparentes, las velocidades del ultrasonido, los módulos dinámicos y los coeficientes de amortiguamiento de la madera de las 22 especies estudiadas. Los estadísticos ahí presentados son la media $(\bar{x})$ y el coeficiente de variación $(C V)$.

La densidad aparente presentó valores en un intervalo entre $338 \mathrm{~kg} / \mathrm{m}^{3}$ y $1147 \mathrm{~kg} / \mathrm{m}^{3}$. La densidad aparente está incorporada en el cálculo del módulo dinámico, así como en el del coeficiente de amortiguamiento. Esta propiedad material está también presente en los índices de rendimiento acústico. La densidad aparente de la madera es resultado de la configuración del tejido anatómico y es particular de cada especie, considerando siempre sus peculiaridades de variación al interior de un árbol, entre individuos al interior de una especie y entre especies. La densidad aparente también es un indicador de la porosidad y del peso, ambos criterios importantes en la apreciación de una madera, por los fabricantes de instrumentos musicales: poca y uniforme porosidad y bajo peso (Buksnowit et al., 2007).

La densidad aparente de la madera es el parámetro que se considera usualmente como buen predictor de sus características mecánicas. En este contexto, una de las peculiaridades más tomadas en cuenta en la calificación de 
una madera es el arreglo de las capas de crecimiento del árbol, en la superficie radial-tangencial del plano leñoso. La homogeneidad del tejido es el indicador visual a considerar, característica que depende principalmente de la regularidad geométrica y del diámetro de las células que conforman el plano leñoso, así como del espesor de la pared celular (Bucur, 2006).

Para el caso de aplicaciones acústicas de resonancia, es decir, instrumentos musicales, el coeficiente de amortiguamiento de la madera se prefiere tan bajo como sea posible. Sin embargo, para fines de aislamiento acústico en edificaciones con madera, se requiere madera con altos índices de amortiguamiento y, para diseño sísmico, es necesario considerar las cualidades de un elemento estructural que disipe la energía provocada por sismos y acontecimientos dinámicos excepcionales.

En la selección de una madera para su empleo como componente de resonancia $\mathrm{y} / \mathrm{o}$ estructural de un instrumento musical es necesario considerar otros factores y características. Las propiedades materiales y los índices de rendimiento acústico son los parámetros de referencia para el análisis de su comportamiento mecánico-acústico. La densidad aparente, la velocidad de onda, en este caso del ultrasonido, el módulo dinámico y el coeficiente de amortiguamiento, son propiedades intensivas de primer orden, es decir que se calculan combinando mediciones de orden cero, como son el peso de la masa del material, las dimensiones espaciales de su volumen y el tiempo.

Estos parámetros de orden cero, se miden directamente sobre probetas de la madera que se pretenden estudiar y durante el periodo que dura el experimento. Su ponderación empleando ecuaciones constitutivas, resulta en parámetros de primer orden, conocidos también como parámetros de ingeniería y utilizados preferentemente en el cálculo estructural de vigas y columnas. La combinación subsecuente, deriva en parámetros de segundo orden, como son los índices de rendimiento acústico, de tal forma que son estos los indicadores necesarios para el diseño y desarrollo de productos (Ashby, 2011).

La tabla 2 presenta los índices de rendimiento acústico. De acuerdo con Bucur (2006), Buksnowit et al. (2007), Yoshikawa (2007) y Yoshikawa y Waltham (2014), y dependiendo de si se trata de placas de resonancia o madera tonal y/o de elementos de resistencia, es decir, madera de bastidor, las magnitudes comparativas de los índices de rendimiento acústico son particularmente necesarios.

La velocidad del ultrasonido explica la capacidad de la madera para trasmitir una onda mecánica, en este caso una onda acústica. Este descriptor varía según la dirección de anisotropía de la madera, de su contenido de humedad y del método de determinación (Roohnia et al., 2011). De acuerdo con los resultados de la tabla 1, la velocidad del ultrasonido no presentó una relación lineal con la densidad aparente de la madera de las 22 especies ensayadas en este trabajo.

La tabla 3 presenta las correlaciones lineales ( $y=a x \pm$ b) y coeficientes de determinación $\left(R^{2}\right)$ entre los parámetros determinados (velocidad del ultrasonido, módulo dinámico, coeficiente de amortiguamiento y coeficiente de eficiencia acústica) y la densidad aparente de la madera. Estas correlaciones corresponden a las figuras 2, 3, 4 y 5 respectivamente. En el mismo contexto y en referencia al análisis subsiguiente, las maderas se asociaron en tres grupos en relación con su densidad aparente: de baja densidad $\left(\varrho_{C H}<516 \mathrm{~kg} / \mathrm{m}^{3}\right)$, de media densidad $\left(516<\varrho_{C H}\right.$ $\left.<692 \mathrm{~kg} / \mathrm{m}^{3}\right)$ y de alta densidad $\left(\varrho_{C H}>692 \mathrm{~kg} / \mathrm{m}^{3}\right)$.

La figura 2 presenta la dispersión de la velocidad del ultrasonido en función de la densidad aparente y se contrasta con datos de Illic (2003). Del análisis de la figura 2 no se infiere ninguna tendencia de la velocidad del ultrasonido cuando la densidad aparente aumenta, resultado que concuerda con la tendencia de los datos de Illic (2003). No obstante, una madera con baja densidad, es uno de los criterios preferentes para su selección en la fabricación de instrumentos musicales. 
TABLA 2. Índices de rendimiento acústico de la madera de 22 especies forestales del estado de Michoacán, México.

\begin{tabular}{|c|c|c|c|c|c|}
\hline No. & Especie & $R_{u s}$ & $C E A_{\text {us }}$ & $I V A_{\text {us }}$ & $I A A_{u s}$ \\
\hline & & $\left(\mathrm{m}^{4} / \mathrm{kg} \mathrm{s}\right)$ & $(\mathrm{m} / \mathrm{s})$ & $(\mathrm{m} / \mathrm{s})$ & $\left(\mathrm{kg} \mathrm{s} / \mathrm{m}^{4}\right)$ \\
\hline 1 & Spathodea campanulata & 10.96 & 1411 & 4.77 & 0.091 \\
\hline 2 & Gyrocarpus americanus & 11.47 & 1925 & 7.53 & 0.087 \\
\hline 3 & Tilia mexicana & 11.89 & 2445 & 10.82 & 0.084 \\
\hline 4 & Enterolobium cyclocarpum & 8.73 & 1209 & 5.42 & 0.115 \\
\hline 5 & Cupressus lindleyi & 9.83 & 1798 & 8.75 & 0.102 \\
\hline 6 & Cedrela odorata & 8.20 & 1264 & 6.54 & 0.122 \\
\hline 7 & Swietenia macrophylla & 8.81 & 1570 & 8.34 & 0.113 \\
\hline 8 & Tabebuia donnell-smithii & 7.20 & 1129 & 6.75 & 0.139 \\
\hline 9 & Dalbergia palo-escrito & 7.53 & 1353 & 8.44 & 0.133 \\
\hline 10 & Tabebuia rosea & 7.50 & 1366 & 8.68 & 0.133 \\
\hline 11 & Fagus mexicana & 7.22 & 1269 & 8.15 & 0.139 \\
\hline 12 & Andira inermis & 5.84 & 889 & 6.36 & 0.171 \\
\hline 13 & Psidium sartorianum & 4.99 & 697 & 5.50 & 0.200 \\
\hline 14 & Juglans pyriformis & 5.65 & 974 & 7.89 & 0.177 \\
\hline 15 & Caesalpinia platyloba & 5.89 & 1104 & 9.10 & 0.170 \\
\hline 16 & Albizia plurijuga & 5.81 & 1100 & 9.28 & 0.172 \\
\hline 17 & Quercus spp. & 6.21 & 1297 & 10.98 & 0.161 \\
\hline 18 & Lysiloma acapulcensis & 4.70 & 810 & 7.89 & 0.213 \\
\hline 19 & Cordia elaeagnoides & 4.70 & 832 & 8.26 & 0.213 \\
\hline 20 & Acosmium panamense & 4.53 & 775 & 7.79 & 0.221 \\
\hline 21 & Tabebuia chrysantha & 4.04 & 666 & 7.29 & 0.247 \\
\hline 22 & Dalbergia granadillo & 3.88 & 645 & 7.40 & 0.258 \\
\hline
\end{tabular}

$R_{u s}=$ Coeficiente de radiación acústica; $C E A_{\text {us }}=$ Coeficiente de eficiencia acústica; $I_{\text {Aus }}$ = Índice de vibración acústica; $I A A_{u s}=$ Índice de anti-vibración acústica.

La figura 3 presenta la dispersión del módulo dinámico en función de la densidad aparente de 22 maderas mexicanas y sus valores se comparan con los datos de Brémaud et al. (2012). Los resultados del módulo dinámico se posicionan proporcionalmente a la densidad aparente de la madera y son similares a los hallados por Brémaud et al. (2012). Un valor alto de módulo dinámico es un buen criterio para la selección de una madera con potencial para su incorporación como elemento constitutivo en un instrumento musical. De tal forma que la madera que combina un valor alto del módulo dinámico con un valor bajo de densidad aparente, se puede reconocer como buena candidata para su empleo en funciones acústicas. 
TABLA 3. Correlaciones y coeficientes de determinación correspondientes a las gráficas presentadas en las figuras $2,3,4$ y 5 de este documento.

\begin{tabular}{|c|c|c|c|}
\hline Figura & Grupo de maderas & $y=a x \pm b$ & $R^{2}$ \\
\hline 2 & Baja densidad & $V_{u s}=6.4246 \rho C H+1723.4$ & 0.34 \\
\hline 2 & Media densidad & $V_{u s}=-1.7184 \rho_{C H}+5515.4$ & 0.26 \\
\hline 2 & Alta densidad & $V_{u s}=-1.4843 \rho_{C H}+6103.4$ & 0.48 \\
\hline 2 & Illic (2003) & $V_{u s}=-0.5128 \rho_{C H}+5123.7$ & 0.03 \\
\hline 3 & Baja densidad & $E_{\text {us }}=41.217 \rho C H-8813.5$ & 0.58 \\
\hline 3 & Media densidad & $E_{\text {us }}=8.303 \rho_{C H}+7098.8$ & 0.20 \\
\hline 3 & Alta densidad & $E_{\text {us }}=8.1549 \rho \mathrm{CH}+13046$ & 0.28 \\
\hline 3 & Brémaud et al. (2012) & $E_{u s}=23.238 \rho C H-2410.6$ & 0.60 \\
\hline 4 & Baja densidad & $\delta_{\text {us }}=-0.000013 \rho \mathrm{CH}+0.0118$ & 0.38 \\
\hline 4 & Media densidad & $\delta_{u s}=0.000004 \rho_{C H}+0.0039$ & 0.27 \\
\hline 4 & Alta densidad & $\delta_{\text {us }}=0.000002 \rho_{C H}+0.0033$ & 0.52 \\
\hline 4 & Brémaud et al. (2012) & $\delta_{u s}=-0.000005 \rho_{C H}+0.0116$ & 0.16 \\
\hline 5 & Baja densidad & $C E A_{u s}=2.0006 \rho C H+915.13$ & 0.06 \\
\hline 5 & Media densidad & $C E A_{u s}=-2.6138 \rho_{C H}+2842.7$ & 0.70 \\
\hline 5 & Alta densidad & $C E A_{u s}=-1.5834 \rho_{C H}+2413.9$ & 0.79 \\
\hline 5 & Brémaud et al. (2012) & $C E A_{\text {us }}=-8.3078 \rho_{C H}+5418.5$ & 0.87 \\
\hline 5 & Sedik et al. (2010) & $C E A_{u s}=-2.7 \rho_{C H}+2729$ & 0.14 \\
\hline
\end{tabular}

Vus = Velocidad del ultrasonido; $\rho c H=$ Densidad aparente; $E_{u s}=$ Módulo dinámico; $\delta_{\text {us }}=$ Coeficiente de amortiguamiento; $C E A_{u s}=$ Coeficiente de eficiencia acústica; $y=a x \pm b=$ Correlación lineal; $R^{2}$ = Coeficiente de determinación.

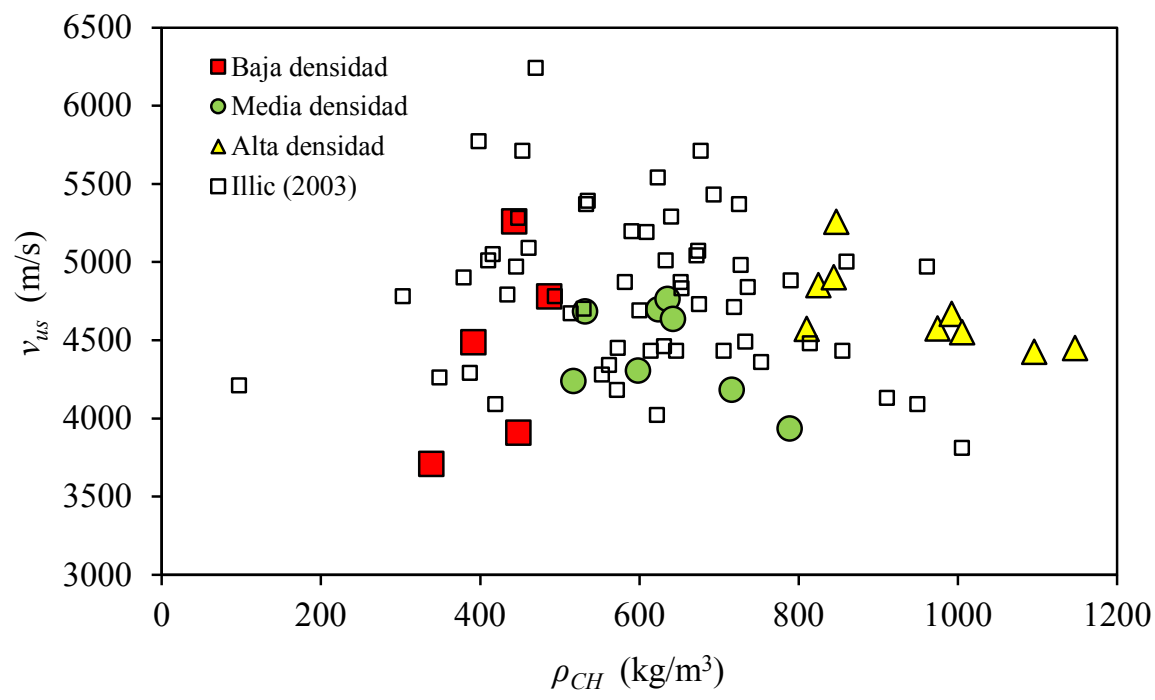

FIGURA 2. Dispersión de la velocidad del ultrasonido $\left(v_{u s}\right)$ en función de la densidad aparente $\left(\varrho_{C H}\right)$ de 22 maderas mexicanas en comparación con los datos de Illic (2003). 


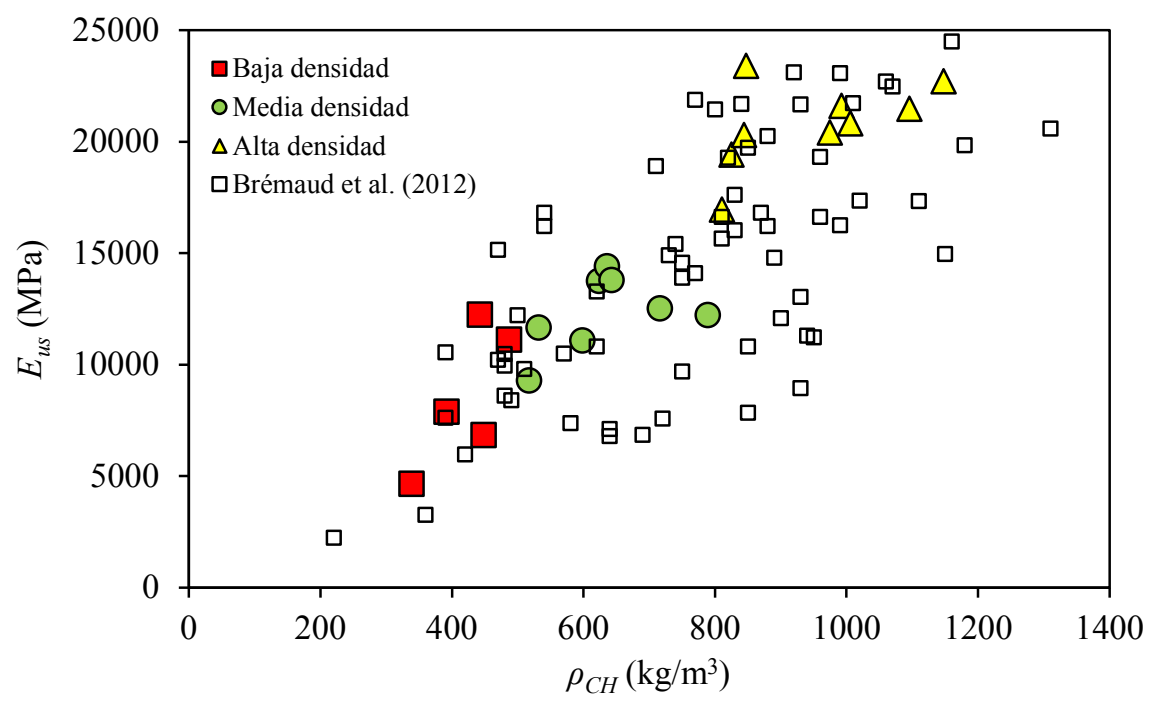

FIGURA 3. Dispersión del módulo dinámico $\left(E_{u s}\right)$ en función de la densidad aparente $\left(\varrho_{C H}\right)$ de 22 maderas mexicanas en comparación con los datos de Brémaud et al. (2012).

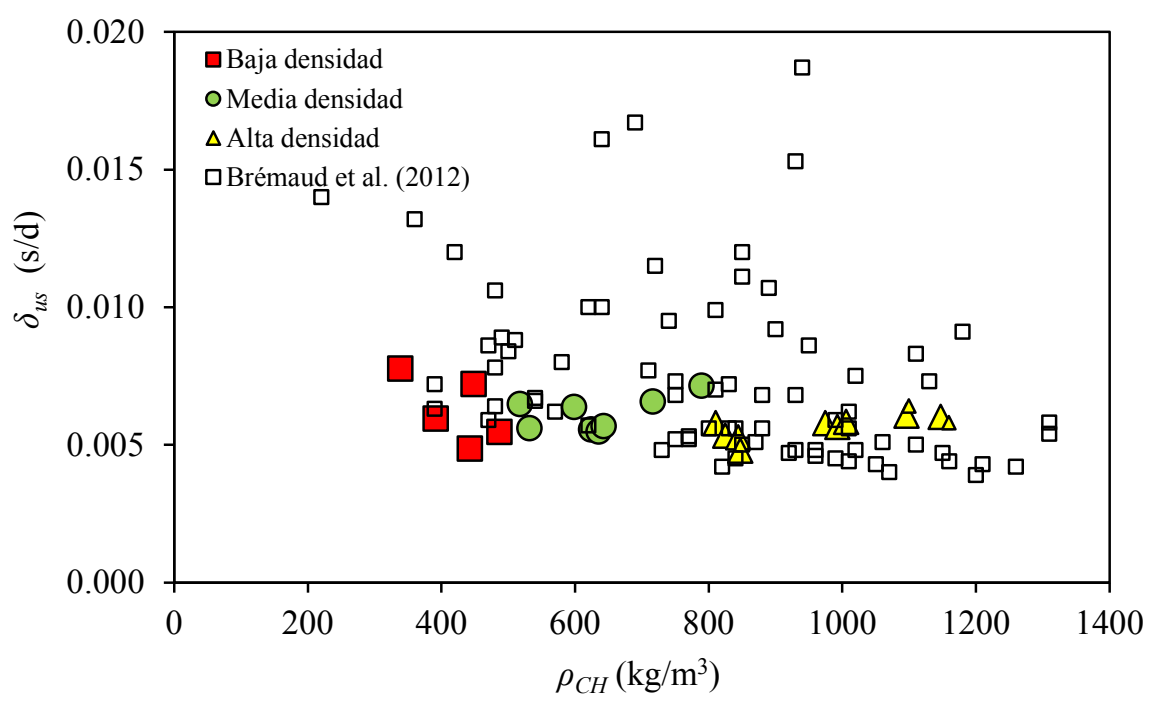

FIGURA 4. Dispersión del coeficiente de amortiguamiento $\left(\delta_{u s}\right)$ en función de la densidad aparente $(\varrho \mathrm{CH})$ de 22 maderas mexicanas en comparación con los datos de Brémaud et al. (2012). 


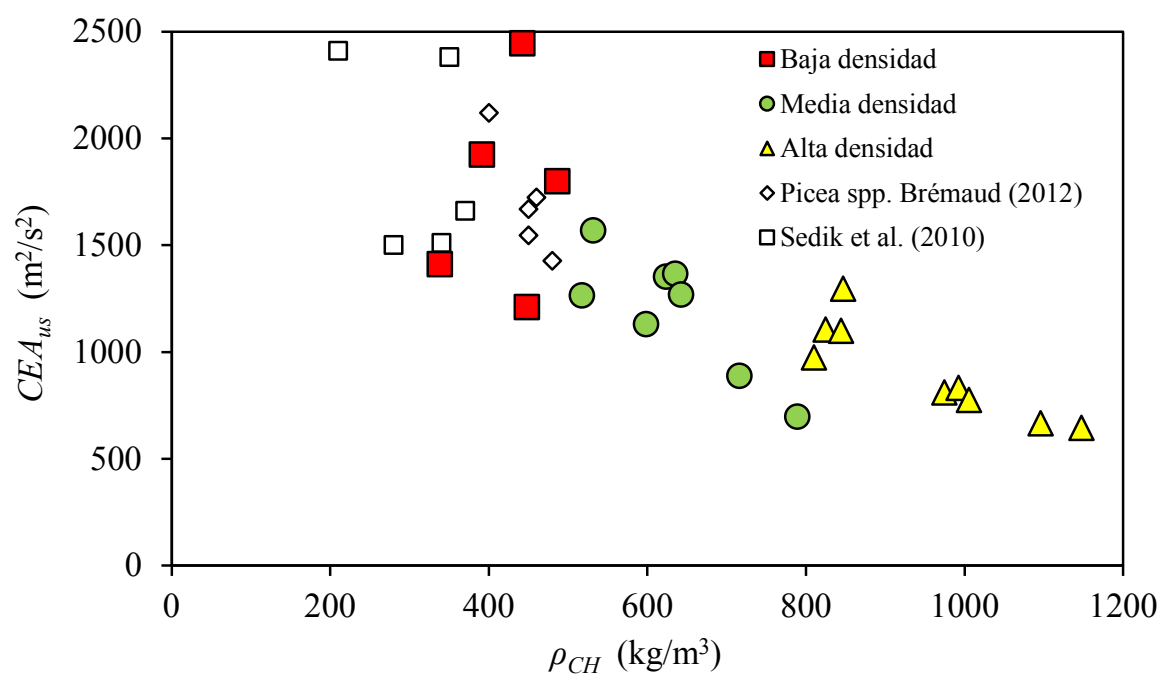

FIGURA 5. Dispersión del coeficiente de eficiencia acústica $\left(C E A_{u s}\right)$ en función de la densidad aparente $\left(\varrho_{C H}\right)$ de 22 maderas mexicanas en comparación con los datos de Picea spp (Brémaud et al. 2012) y de Sedik et al. (2010).

El coeficiente de amortiguamiento explica la cantidad de energía acústica que una pieza de madera puede disipar y es igualmente una expresión de la propiedad que poseen los materiales sólidos para transformar energía mecánica en calor, mediante el fenómeno de frotamiento interno cuando están sujetos a esfuerzos dinámicos ocasionando pequeñas deformaciones elásticas. Este descriptor es derivado de la medición del decremento temporal en la amplitud de una señal acústica (Brancheriau, Kouchade, y Brémaud, 2010).

El coeficiente de amortiguamiento de las maderas de baja, media y alta densidad aparente es constante en relación con la magnitud de la densidad aparente (Fig. 4). Sus valores son similares a los encontrados por Brémaud et al. (2012) y en comparación con algunas especies, son bajos para la densidad aparente correspondiente. Sin embargo, se observa que el amortiguamiento de las maderas de alta densidad aparente presenta bajo amortiguamiento. De tal forma que estas se adecuan al axioma: a menor amortiguamiento, mayor resonancia y menor extinción del sonido, de acuerdo con Buksnowit et al. (2007), Yoshikawa (2007) y Yoshikawa y Waltham (2014).
En síntesis, un buen elemento estructural para un instrumento musical requiere básicamente altos valores de módulo dinámico, bajos valores de densidad aparente y de amortiguamiento y altas relaciones de anisotropía entre las direcciones longitudinal y transversal de la madera (Se Golpayegani et al., 2012).

Brancheriau et al. (2006), proponen para madera de buena calidad acústica un intervalo para el coeficiente de amortiguamiento entre 0.0082 y 0.0126 . De aquí, que las maderas investigadas presentan un coeficiente de amortiguamiento menor lo que las posiciona probablemente como madera con buena calidad acústica.

Las funciones principales de un componente de resonancia son minimizar las vibraciones y maximizar la resonancia. Estas funciones son explicadas por el coeficiente de radiación acústica, el cual interpreta la capacidad de radiación del sonido de un elemento de resonancia (Yoshikawa, 2007 y Yoshikawa y Waltham, 2014).

El coeficiente de radiación acústica caracteriza la transmisión de la vibración de la madera y representa la respuesta pico de una señal acústica (Brémaud, 2012b). Igualmente describe la velocidad con la que la vibración de 
un cuerpo es amortiguada (Wegst, 2006). Para componentes de resonancia, se recomienda un alto coeficiente de radiación acústica (Rujinirun, Phinyocheep, Prachyabrued y Laemsak 2005). De tal forma, que representa una medida del amortiguamiento de ondas acústicas, particularmente en instrumentos musicales de cuerdas (Spycher et al. 2008).

La figura 5 detalla la dispersión del coeficiente de eficiencia acústica en función de la densidad aparente y compara los resultados de esta investigación con los datos de Picea spp. publicados por Brémaud et al. (2012) y Sedik et al. (2010). Los resultados de las maderas aquí estudiadas siguen la tendencia de los datos de los autores citados, y sus correlaciones lineales presentan fuertes coeficientes de determinación. Así, las maderas de baja densidad aparente presentan mejores coeficientes de eficiencia acústica comparativamente con las especies de altas densidades.

A manera de prospección, seis de las ocho maderas mexicanas de media densidad, son utilizadas principalmente en componentes con una función de resistencia estructural, por ejemplo, brazo, puente, costilla y diapasón, así como en la caja de resonancia de guitarras, charangos y vihuelas (Guridi y García, 1997). En contraste, no se han encontrado menciones de uso de $A$. inermis y $P$. sartorianum en instrumentos musicales.

Las coordenadas de las maderas con baja densidad aparente, aquí estudiadas y mostradas en la figura 5, las posicionan congruentemente en el mismo rango que las de Picea spp. descritas por Brémaud et al. (2012). Igualmente, es el caso para las especies referidas por Sedik et al. (2010).

Con la misma tendencia, pero de manera diferente, las maderas con densidades medias y altas, aparentemente siguen la tendencia de menor coeficiente de eficiencia acústica al aumento de la densidad aparente. De aquí, que maderas con densidades altas y medias, pueden amortiguar de manera más eficiente las vibraciones, y en consecuencia, el sonido de un instrumento es menos brillante y se opacará rápidamente. Este indicador es útil entonces en componentes donde es necesario controlar la resonancia.

En contraste, y a manera de comparación práctica del análisis de los resultados presentados en la figura 5 , cuatro de las maderas de baja densidad: S. campanulata, $G$. americanus, T. mexicana y C. lindleyi, son utilizadas en tapas para guitarras (Guridi y García, 1997). Estas tienen altos coeficientes de radiación acústica y son comparables con las documentadas por Sedik et al. (2010), y son de densidades similares a las del grupo de Picea spp., todas se utilizan generalmente en la fabricación de instrumentos musicales, puesto que trasforman fácilmente la energía de vibración, en sonido.

En cambio, la madera de E. cyclocarpum, no obstante que posee un alto coeficiente de radiación acústica, es usada principalmente en artesanías y muebles, también de manufactura artesanal. Sin embargo, y de acuerdo con los resultados de la figura 5, se puede presumir, que un componente de un instrumento musical elaborado con esta madera, probablemente se comportará como un buen resonador. Una correcta aplicación de tecnologías de estabilizado de la madera, de su maquinado y su terminado de superficie, mejorarán aún más sus aptitudes acústicas.

En síntesis, se puede proponer que los valores del índice de rendimiento obtenidos para las maderas de baja densidad G. americanus, T. mexicana y C. lindleyi, convierten eficientemente la energía de vibración en energía sónica.

El índice de vibración acústica $\left(I V A_{\text {us }}\right)$ representa la capacidad de transmitir vibraciones, mientras que el índice de anti-vibración acústica $\left(I A A_{u s}\right)$ es una medida de la resistencia a la vibración (Yoshikawa, 2007). En la figura 6, la recta $I V A_{u s}=-50.5 L A A_{u s}+11.4$ (Yoshisawa, 2014), corresponde al criterio de selección para placas de resonancia. La combinación de coordenadas de los índices IV $A_{u s}$ e $I A A_{u s}$ de las maderas que se acerquen a esta tendencia, probablemente poseen aptitudes para ser empleadas en instrumentos musicales, particularmente en elementos donde la función de resonancia es prioritaria. 


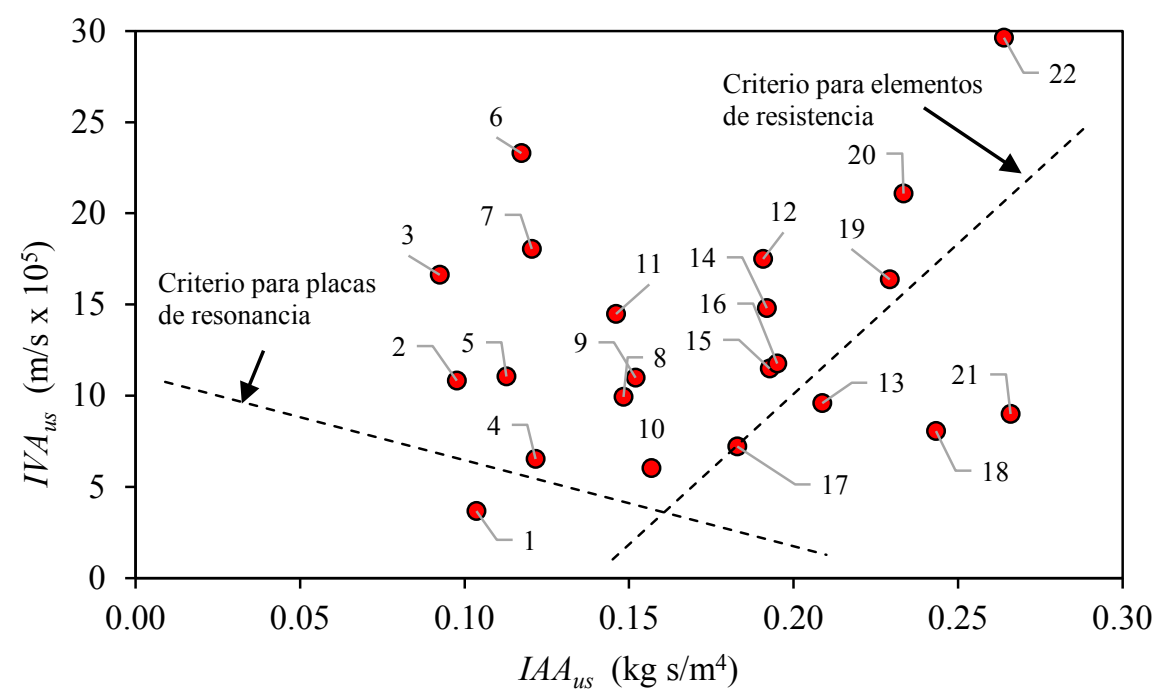

FIGURA 6. Dispersión del índice de vibración acústica $\left(I V A_{u s}\right)$ en función del índice de anti-vibración acústica $\left(I A A_{u s}\right)$ de 22 maderas mexicanas y los criterios de selección para placas de resonancia y para elementos de resistencia, de acuerdo con Yoshisawa (2014). La leyenda coincide con la numeración de especies de la Tabla 1.

En la figura 6, la recta para elementos de resistencia $I V A_{u s}=143 I A A_{u s}-18.9$ (Yoshisawa, 2014), corresponde al criterio de selección de elementos de resistencia. Al igual que el criterio para placas de resonancia, la combinación de coordenadas de los índices IV $A_{u s}$ y $L A A_{u s}$ de las maderas que se acerquen a esta tendencia, probablemente poseen aptitudes para ser empleadas en instrumentos musicales, pero en este caso, en situaciones en donde la función principal es soportar cargas y funcionar como soporte estructural.

Estos criterios son derivados de investigación con maderas utilizadas en la elaboración de instrumentos musicales, y probablemente son convenientes para la selección de las maderas mexicanas estudiadas. Sin embargo, estas guías se podrían ajustar si las rectas presentadas en la figura 6 , se determinan con maderas regionales o endémicas y valoradas en usos acústicos. Para el caso de México, de la lista de especies presentadas en la Tabla 1, seis de ellas no tienen ninguna referencia bibliográfica sobre su uso en instrumentos musicales: $A$. inermis, P. sartorianum, C. platyloba, A. plurijuga, L. acapulcensis y C. elaeagnoides.

\section{CONCLUSIONES}

Un criterio de selección para una madera con aptitudes para ser utilizada en la fabricación de instrumentos musicales es la combinación de una baja densidad aparente, un alto módulo dinámico y un bajo coeficiente de amortiguamiento.

No obstante, la selección de una madera para su empleo como componente estructural de un instrumento musical, necesita tomar en consideración otros factores y características. Desde el punto de vista de la calidad o rendimiento acústicos, un solo índice no es suficiente para clasificar una madera. De tal forma que es recomendable considerar el coeficiente de radiación acústica, el coeficiente de eficiencia acústica, el coeficiente de radiación acústica, el índice de vibración acústica y el índice de anti-vibración acústica.

Las propiedades materiales y los índices de rendimiento acústico de las veintidós maderas mexicanas, son particulares a cada una de ellas. Si se comparan con los resultados publicados en la bibliografía, probablemente la madera de estas especies se valorice para su empleo en funciones donde el rendimiento acústico es importante. Para redondear esta conclusión, es recomendable realizar 
estudios de maquinado y terminado de superficie para tener una visión completa de su posible aprovechamiento como material de ingeniería en la elaboración de instrumentos musicales.

\section{REFERENCIAS}

Ashby, M. F. (2011). Materials selection in mechanical design (4a ed). Burlington: Elsevier.

Barajas M., J., \& León G, C. (1989). Anatomía de maderas de México: Especies de una selva caducifolia. México: Universidad Nacional Autónoma de México.

Brancheriau, L., Baillères, H., Détienne, P., Gril, J., \& Kronland, R. (2006). Key signal and wood anatomy parameters related to the acoustic quality of wood for xylophone-type percussion instruments. Journal of Wood Science, 52(3), 270-273. doi: 10.1007/s10086-005-0755-2

Brancheriau, L., Kouchade, C., \& Brémaud, I. (2010). Internal friction measurement of tropical species by various acoustic methods. Journal of Wood Science, 56(5), 371-379. doi: 10.1007/s10086-010$1111-8$

Brémaud, I. (2012a). Acoustical properties of wood in string instruments soundboards and tuned idiophones: Biological and cultural diversity. Journal of the Acoustical Society of America, 131(1), 807-818. doi: $10.1121 / 1.3651233$

Brémaud, I. (2012b). What do we know on "resonance wood" properties? Selective review and ongoing research. Proceedings of the Acoustics 2012 (pp. 2759-2764). Nantes Conference.

Brémaud, I., El Kaïm, Y., Guibal, D., Minato, K., Thibaut, B., \& Gril, J. (2012). Characterization and categorization of the diversity in viscoelastic vibrational properties between 98 wood types. Annals of Forest Science, 69(3), 373-386. doi: 10.1007/s13595-011-0166-z

Bucur, V. (2006). Acoustics of wood (2a ed.). Berlín: Springer-Verlag.

Buksnowitz, C., Teischinger, A., Müller, U., Pahler, A., \& Evans, R. (2007). Resonance wood [Picea abies (L.) Karst.]. Evaluation and prediction of violinmakers' quality grading. Journal of the Acoustical Society of America, 121(4), 2384-2395. doi: 10.1121/1.2434756

Dietsch, P., \& Köhler, J. (2010). Assessment of timber structures. COST Action E55. Modelling of the performance of timber structures. European Science Foundation. Herzogenrath: Shaker Verlag.

Fouilhé, E., Houssay, A., \& Brémaud, I. (2012). Dense and hard woods in musical instrument making: comparison of mechanical properties and perceptual "quality" grading. Proceedings of the Acoustics 2012 (pp. 2689-2694). Nantes Conference.
Guridi G., L.I., \& García L, A. (1997). Las maderas en los instrumentos musicales de cuerda de Paracho. Morelia: Secretaría de Difusión cultural-Universidad Michoacana de San Nicolás de Hidalgo.

Gutiérrez C., L., \& Dorantes L, J. (2007). Especies forestales de uso tradicional del Estado de Veracru₹: México: Conafor-Conacyt-UV.

Ilic, J. (2003). Dynamic MOE of 55 species using small wood beams. Holz als Rob-und Werkstoff, 61(3), 167-172. doi: 10.1007/s00107003-0367-8

International Organization for Standardization [ISO]. (2014). ISO 13061-1:2014 Physical and mechanical properties of wood. Test methods for small clear wood specimens. Part 1: Determination of moisture content for physical and mechanical tests. International Organization for Standardization. Geneva: ISO

Obataya E., Ono T., \& Norimoto, M. (2000). Vibrational properties of wood along the grain. Journal of Materials Science, 35(12), 2993-3001. doi:10.1023/A:1004782827844

Ono, T., \& Norimoto, M. (1983). Study on Young's Modulus and Internal Friction of Wood in Relation to the Evaluation of Wood for Musical Instruments. Japanese Journal of Applied Physics, 22(4), 611-614. doi: 10.1143/JJAP.22.611

Ono, T., \& Norimoto, M. (1984). On physical criteria for the selection of wood for soundboards of musical instruments. Rheologica Acta, 23(6), 652-656. doi: 10.1007/BF01438805

Ono, T., \& Norimoto, M. (1985). Anisotropy of Dynamic Young's Modulus and Internal Friction in Wood. Japanese Journal of Applied Physics, 24(8), 960-964. doi: 10.1143/JJAP.24.960

Roohnia, M., Tajdini, A., \& Manouchehri, N. (2011). Assessing wood in sounding boards considering the ratio of acoustical anisotropy. NDT \& E International, 44(1), 13-20. doi: 10.1016/j.ndteint.(2010).09.001

Rujinirun, C., Phinyocheep, P., Prachyabrued, W., \& Laemsak, N. (2005). Chemical treatment of wood for musical instruments. Part I: acoustically important properties of wood for the Ranad (Thai traditional xylophone). Wood Science and Technology, 39(1), 77-85. doi: 10.1007/s00226-004-0275-z

Se Golpayegani, A., Brémaud, I., Gril, J., Thevenon, M-F., Arnould, O., \& Pourtahmasi, K. (2012). Effect of extractions on dynamic mechanical properties of white mulberry (Morus alba). Journal of Wood Science, 58(2), 153-162. doi: 10.1007/s10086-011-1225-7

Sedik, Y., Hamdan, S., Jusoh, I., \& Hasan, M. (2010). Acoustic Properties of Selected Tropical Wood Species. Journal of Nondestructive Evaluation, 29(1), 38-42. doi: 10.1007/s10921-010-0063-7

Silva G., J. A., Fuentes T., F. J., Rodríguez A., R., Torres A., P. A., Lomelí R., M. A., Ramos Q., J., Waitkus, C., \& Richter, G. H. (2010). 
Fichas de propiedades tecnológicas y usos de maderas nativas de México e importadas. México: Comisión Nacional Forestal.

Smardzewski, J., Batko, W., Kamisiński, T., Flach, A., Pilch, A., Dziurka, D., Mirski, R., Roszyk, E., \& Majewski, A. (2014). Experimental study of wood acoustic absorption characteristics. Holzforschung, 68(4), 467-476. doi: 10.1515/hf-2013-0160

Sotomayor C., J. R., Guridi G., L. I., \& García M, T. (2010). Características acústicas de la madera de 152 especies mexicanas. Velocidad del ultrasonido, módulo de elasticidad, índice material y factor de calidad. Base de datos. Investigación e Ingeniería de la Madera, 6(1), 3-32.

Spycher, M., Schwarze, F. W. M. R., \& Steiger, R. (2008). Assessment of resonance wood quality by comparing its physical and histological properties. Wood Science and Technology, 42(4), 325-342. doi: $10.1007 /$ s00226-007-0170-5

Tamarit U., J.C., \& López T., J. L. (2007). Xilotecnología de los principales árboles tropicales de México. Libro técnico No. 3. Tlahuapan. Puebla: Inifap-CIR Golfo Centro.

Traoré, B., Loïc, B., Perré, P., Stevanovic, T., \& Diouf, P. (2010). Acoustic quality of vène wood (Pterocarpus erinaceus Poir.) for xylophone instrument manufacture in Mali. Annals of Forest Science, 67(8), 815p1-815p7. doi: 10.1051/ forest/(2010)054
Wegst, U. G. K. (2006). Wood for sound. American Journal of Botany, 93(10), 1439-1448. doi: 10.3732/ajb.93.10.1439

Yoshikawa, S. (2007). Acoustical classification of woods for string instruments. Journal of the Acoustical Society of America, 122(1), 568573. doi: $10.1121 / 1.2743162$

Yoshikawa, S., \& Waltham, C. (2014). Woods for Wooden Musical Instruments. In: Proceeding of ISMA 2014 (pp. 281-286). Le Mans, France.

Manuscrito recibido el 3de agosto de 2016

Aceptado el 1 de marzo de 2018

Publicado el 1 de noviembre de 2018

\section{Este documento se debe citar como:}

Sotomayor C., J. R., \& Villaseñor A., J. M. Propiedades materiales e índices de rendimiento acústico de veintidós maderas mexicanas. Determinación por ultrasonido. Madera y Bosques, 24(3), e2431132. doi: 10.21829/myb.2018.2431132

Madera y Bosques por Instituto de Ecología, A.C. se distribuye bajo una Licencia Creative Commons Atribución-NoComercialCompartirlgual 4.0 Internacional. 